\title{
Trends in Bragg Grating Technology for Optical Fiber Sensor Applications
}

\author{
Hartmut Bartelt \\ Institute of Photonic Technology (IPHT) \\ Albert-Einstein-Str. 9, 07745 Jena, Germany \\ hartmut.bartelt@ipht-jena.de
}

\begin{abstract}
Keywords: Fiber Bragg Gratings, Bragg Grating Sensors, Spectrally Encoded Fiber Sensor Systems
\end{abstract}

\begin{abstract}
Fiber Bragg gratings have found widespread and successful applications in optical sensor systems, e. g. for temperature, strain or refractive index measurements. Such sensor elements are fiber integrated, are applicable under harsh environmental conditions, and can be easily multiplexed. In order to further extend the field of applications, there is a great interest in specifically adapted Bragg gratings, in Bragg grating structures with increased stability, or in the use of special fiber types for grating inscription. The paper discusses such specific concepts for grating inscription, covers novel aspects of fiber gratings in small diameter fibers or in fiber tapers, of gratings in pure silica fibers without UV sensitivity, of grating inscription in different microstructured fibers or photonic crystal fibers, and investigates the concept of femtosecond inscription and the extension of the Bragg reflection wavelengths down to the visible range.
\end{abstract}

\section{Introduction}

Fiber Bragg gratings (FBGs) have become standard elements in the development of new types of fiber-optical sensors and sensor systems [1-2]. Standard techniques for making such fiber Bragg gratings are the phase mask recording technique and the interferometric recording technique. Such gratings have been developed also for use in harsh environments like high temperatures [3]. Multiple gratings are of specific interest in sensor systems in order to allow quasi-distributed sensing and for achieving sensor multiplexing. So-called draw tower gratings, which are recorded by single pulses during the fiber drawing process, have been developed especially for this purpose [4-7]. These examples show the need for specific developments adapted to the needs of fiber sensor applications. Further special developments should therefore extend the capabilities to apply fiber Bragg gratings for additional sensing applications. Below we will discuss such developments for improved and extended sensor applications such as:

- $\quad$ FBGs in small diameter fibers;

- $\quad$ FBGs in fiber tapers;

- $\quad$ FBGs for short wavelengths;

- $\quad$ FBGs in microstructured fibers.

\section{Fiber Bragg Gratings in Small Diameter Fibers}

Due to their small diameters, optical fibers are attractive sensor elements to be embeded in structural materials. Examples are fiber-reinforced thermoplastic composites. By embedding sensors such as fiber Bragg gratings into the material it becomes possible to monitor the structural behaviour during production and/or operation. For such applications one should avoid any disturbance of the composite structure by the sensor element. Therefore, smaller diameters of optical fibers than those typically used in communication applications are desirable. Special fibers for this purpose have been fabricated with fiber diameters of $80 \mu \mathrm{m}$ for grating inscription during the fiber drawing process. It has been shown that even for such small diameter fibers efficient 
gratings can be achieved that show similar properties compared to gratings in conventionally sized fibers with a diameter of $125 \mu \mathrm{m}[8]$.

\section{Fiber Bragg Gratings in Fiber tapers}

The interaction of the guided light in an FBG with the surrounding material can be used for a refractive sensor. In a more complex setup with metallized fibers also the effect of generating plasmonic waves can modify the reflection properties for FBGs in a sensitive way. Such effects require, however, an efficient overlapping of the guided light with the surrounding analyte. For this purpose, fibers with a small core diameter are needed with a reasonable extension of the evanescent wave in the medium to be measured. One possible approach is the use of side-polished fibers. In this case, good overlapping of the guided light is obtained only in one direction, which is a limiting factor for the achievable sensitivity. Optimized sensitivity can therefore be expected for tapered fiber structures with extremely small fiber cores. Typical techniques for making fiber tapers are drawing or etching technologies.

The concept for a tapered sensor probe is shown in Fig. 1. In the short, tapered fiber end, a fiber Bragg grating structure is inscribed. Additional layers of metal and high-refractive-index tantalum pentoxide can be applied to achieve increased field amplitudes at the core boundary and to shift the surface plasmon resonance to smaller refractive index values of the surrounding medium (adapted to the refractive index of water). This FBG is combined with a second FBG in the conventional part of the fiber for temperature measurements and temperature compensation. This FBG sensor element can be used as a refractive sensor, where the refractive index value can be modified e.g. by specific biological binding reactions. Specific sensor elements have been made by an etching process achieving sensor tip diameters of a few micrometers and down to submicrometer dimensions. A result from a sensor probe with a diameter of $3 \mu \mathrm{m}$ is shown in Fig. 1.

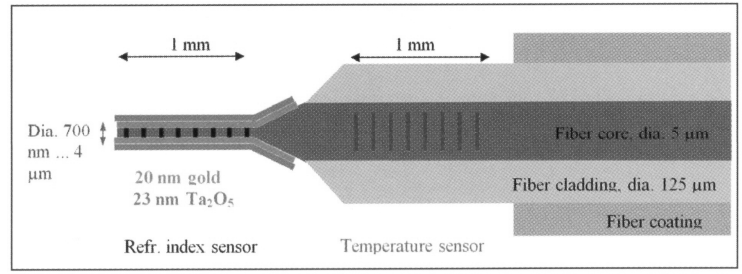

Fig. 1. Concept of an etched fiber taper with FBGs (top), shift of the FBG reflection wavelength with refractive index of a surrounding analyte (right)

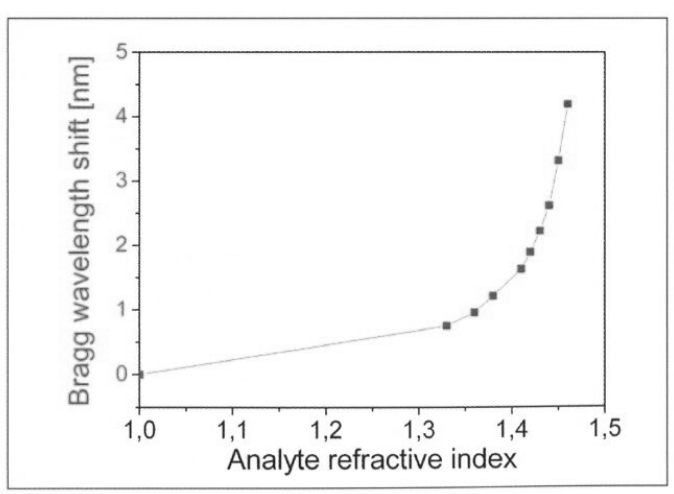

In a similar way it is possible to use tapers made by a taper drawing process (Fig. 2). For Gedoped fiber cores, the diameter of the UV-sensitive region is decreased during the tapering process. Therefore the possible reflectivities achieved by UV inscription are limited for such small tapers. Starting with a conventional fiber $(125 \mu \mathrm{m}$ outer diameter, $3.9 \mu \mathrm{m}$ core diameter $)$, taper diameters down to about $65 \mu \mathrm{m}$ enabled the production of fiber Bragg gratings with reflectivities of more than $30 \%$. For tapers with smaller diameters, a femtosecond inscription technique proved to be more efficient than the UV inscription technique with nanosecond pulses. The strain properties of such fiber tapers are highly diameter-dependent with a proportionality of $1 / \mathrm{r}^{2}$. These elements can therefore be used as sensitive force sensors down to a range of $1 \mu \mathrm{N}$.

\section{Fiber Bragg Gratings for Short Wavelengths}

The typical wavelengths for fiber grating applications lie in the near infrared region, corresponding to the wavelengths mostly used in waveguide optics. In order to cover new application fields, there is a growing interest to have available fiber Bragg gratings also for shorter wavelengths than those 
of the communication window wavelengths, especially in the visible wavelength range. However, the fabrication of such gratings presents special challenges due to the requirement of very small grating periods in the range of a few $100 \mathrm{~nm}$.

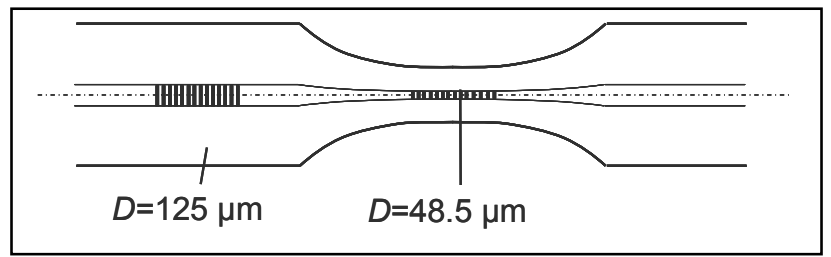

Fig. 2 Concept of a drawn fiber taper with FBGs (top), achieved reflection spectra in the near infrared wavelength region (right)

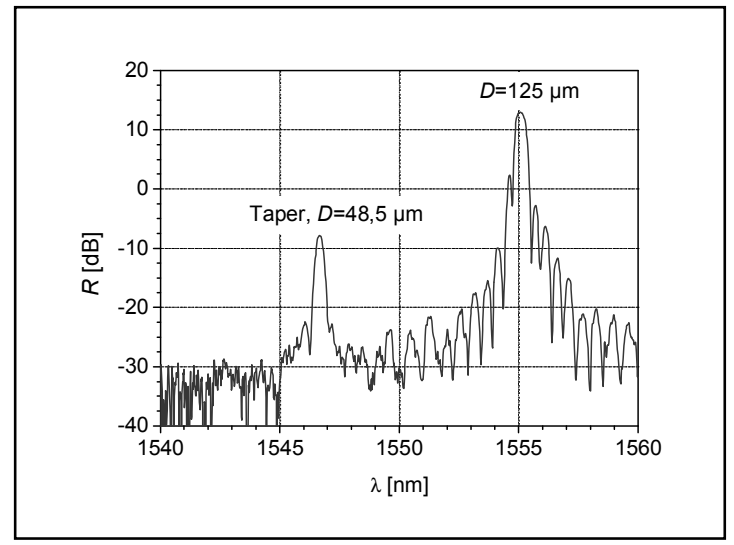

\section{Fiber Bragg Gratings for Short Wavelengths}

The typical wavelengths for fiber grating applications lie in the near infrared region corresponding to the mostly used wavelengths in waveguide optics. In order to cover new application fields, there is a growing interest to have available fiber Bragg gratings also for shorter wavelengths than the communication window wavelengths, especially in the visible wavelength range. However, the fabrication of such gratings presents special challenges due to the requirement of very small grating periods in the range of a few $100 \mathrm{~nm}$.

We use a Talbot interferometer for inscribing Bragg gratings, where we can change the spatial frequency of the Bragg gratings by a variation of the angle $\delta_{\mathrm{FBG}}$ of the interferometer mirrors. The Bragg wavelength $\lambda_{\mathrm{B}}$ then is a function of the Bragg period $\Lambda_{\mathrm{FBG}}$, the effective refractive index $\mathrm{n}_{\mathrm{eff}}$ in the fiber and the UV laser source wavelength $\lambda_{\mathrm{UV}}$, according to:

$$
\lambda_{B}=2 \cdot n_{\text {eff }} \cdot \Lambda_{F B G}=\frac{n_{\text {eff }} \cdot \lambda_{U V}}{\sin \vartheta_{F B G}}
$$

By increasing the angle we have achieved to make FBGs down to a reflection wavelength of about $475 \mathrm{~nm}$ (Fig. 3) in a non-polarization-maintaining single-mode fiber. A reflectivity of about $80 \%$ with a spectral width smaller than $0.3 \mathrm{~nm}$ was obtained with an exposure time of $10 \mathrm{~min}$. This wavelength value still does not represent the experimental limit of the setup, but was given by the available light source for observation (xenon light source with a band edge at $460 \mathrm{~nm}$ ). A theoretical limit considering the recording wavelength used, the experimental setup and the increasing Fresnel reflections has been derived for a wavelength of about $360 \mathrm{~nm}$.

The impressingly high reflectivities, even for small fiber grating structures of about $200 \mathrm{~nm}$, can be explained by the coupled wave theory. The required refractive index modulation of a grating is given by $[10,11]$ :

$$
\Delta n_{A C}=\frac{\operatorname{arctanh}(\sqrt{R}) \cdot \lambda_{B} \cdot\left(\lambda_{B}^{2}+\pi^{2} d^{2} N A^{2}\right)}{\pi^{3} d^{2} l_{F B G} N A^{2}}
$$

For a constant grating length $1_{\mathrm{FBG}}$ and a constant fiber diameter $d$, a reduced index modulation is still sufficient for good reflectivity. The mode overlap factor increases with shorter wavelengths. At the same time, also the number of grating periods increases within the constant grating length. Both factors are helpful to achieve good reflectivity values. 


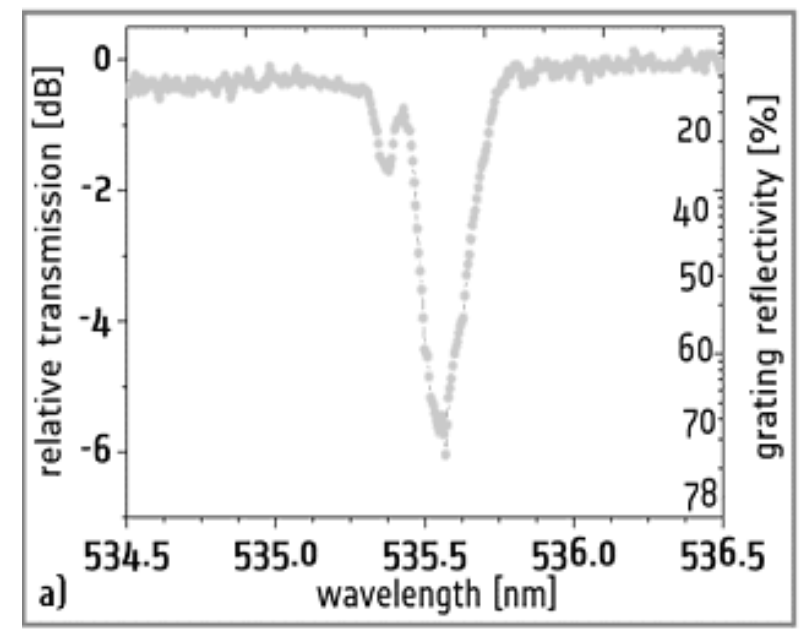

Fig. 3. FBG with short reflection wavelength in the visible range

\section{Fiber Bragg Gratings in Microstructured Fibers (Photonic Crystal Fibers)}

Microstructured fibers or Photonic Crystal Fibers (PCFs) with holey parts in the core and cladding regions offer new possibilities for modifying the transmission properties of optical fibers. Usually, such fibers do not require doping of the fiber core for achieving guiding properties. Therefore the question arises how to make FBGs in such fibers [9, 10, 11].

As an obvious way, it is possible to make such microstructured fibers with a specifically Gedoped fiber core. In this case the doping would be not used for achieving guiding properties, but for obtaining a UV sensitivity. The holey structure may eventually cause reflections and scattering effects. Such effects could make the inscription of efficient gratings difficult. But experimental results show, that indeed high reflectivivties can be achieved with conventional interferometric recording techniques.

If no UV- sensitivity is provided in the fiber core, then other techniques have to be used for writing FBGs. One option is the inscription with femtosecond laser pulses. We have therefore investigated the same microstructured fiber for FBG inscription with a femtosecond laser [11, 12]. Usually, such lasers are used in the near infrared wavelength region. In our case we have used femtosecond laser pulses at a wavelength of $262 \mathrm{~nm}$ in order to inscribe FBGs with high spatial resolution. At this wavelength, effects of the UV sensitivity also can be expected. As shown in Fig. 4 it is possible to achieve good reflection properties both without and with hydrogen loading. The $3 \mathrm{~dB}$-bandwidth is typically larger (about $0.4 \mathrm{~nm}$ ) compared to gratings inscribed in Ge-doped cores with nanosecond excimer laser pulses.

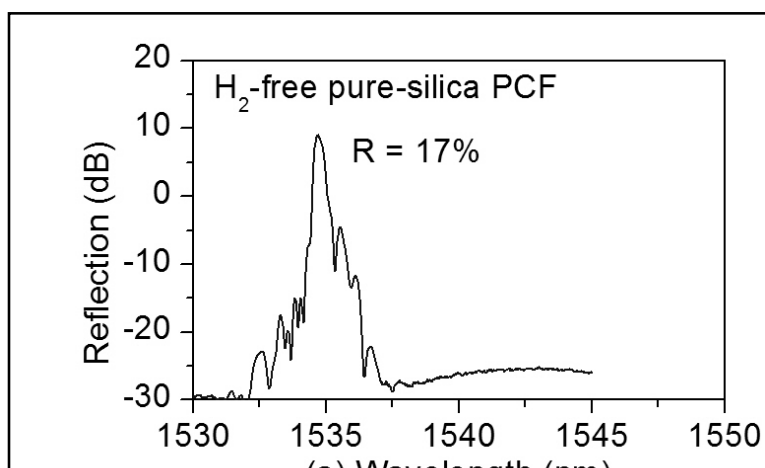

(a) Wavelength $(\mathrm{nm})$

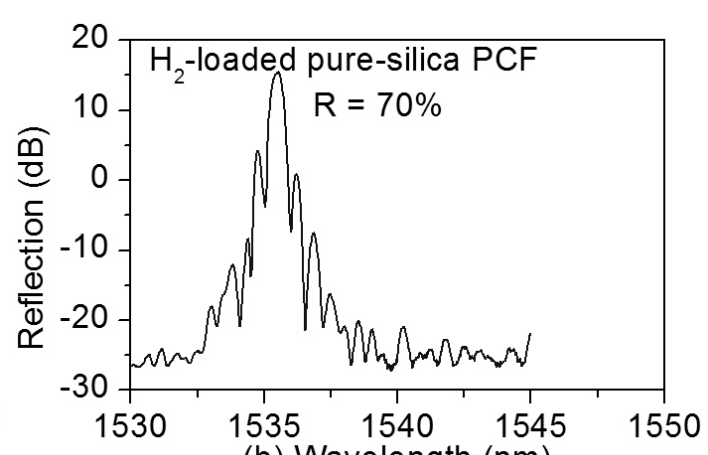

(b) Wavelength (nm)

Fig. 4. FBG reflection spectra for gratings inscribed with femtosecond pulses in pure silica PCFs 


\section{Conclusions}

Several techniques and concepts have been discussed which will allow to extend further the field of applications of FBGs. The great flexibility of such fiber-integrated sensor probes with regard to fiber types and dimensions underlines their growing potential in sensing applications. Additionally, such flexibility will be of interest for further applications in information technology or for fiber lasers.

\section{Acknowledgments}

Contributions by Yiping Wang, Eric Lindner, Manfred Rothhardt, Martin Becker, Michael Kautz, Stefan Demmler, Torsten Wieduwilt, Reinhardt Willsch and Eli Voet in the field of new types of fiber Bragg gratings are gratefully acknowledged. This work was supported by the Alexander von Humboldt Foundation and the Thuringian Ministry of Education and Cultural Affairs.

\section{References}

[1] O.H. Hill, G. Meltz: Fiber Bragg Grating Technology Fundamentals and Overview. Journal of Lightwave Technology 15(8) (1997), pp. 1263-1276.

[2] Andreas Othonos, Kyriacos Kalli: Fiber Bragg Gratings: Fundamentals and Applications in Telecommunications and Sensing. Bd. 1. Artech House (1999).

[3] H. Bartelt, K. Schuster, S. Unger, C. Chojetzki, M. Rothhardt, I. Latka: Single-pulse fiber Bragg gratings and specific coatings for use at elevated temperatures. Appl. Optics 46 (17) (2007), pp. 3417-3424.

[4] C.G. Askins, T.-E. Tsai, G.M. Williams, M.A. Putnam, M. Bashkansky and E.J. Friebele: Fiber Bragg reflectors prepared by a single excimer pulse. Opt. Lett. 17(11) (1992), pp. 833-835.

[5] J.-L. Archambault, L. Reekie, and P.St.J. Russell: 100\% reflectivity Bragg reflectors produced in optical fibres by single excimer laser pulses. Electron. Lett. 29(5) (1993), pp. 453-455.

[6] L. Dong, J.L. Archambault, L. Reekie, P.St.J. Russell, and D.N. Payne: Single pulse Bragg gratings written during fibre drawing. Electron. Lett. 29(17) (1993), pp. 1577-1578.

[7] M. Rothhardt, C. Chojetzki, H.R. Mueller: High mechanical strength single-pulse draw tower gratings. Proc. SPIE. Vol. 5579, Part A (2004), pp. 127-135.

[8] E.J. Voet, G. Luyckx, I. De Baere, J. Degrieck, J. Vlekken and E. Jacobs, H. Bartelt: High Strain monitoring during Fatigue Loading of Thermoplastic Composites using imbedded draw Tower Fibre Bragg Grating Sensors. Paper Nr. C-4: L16, Proceedings CIMTEC 2008.

[9] L.B. Fu et al.: Femtosecond laser writing Bragg gratings in pure silica photonic crystal fibres. Elect. Lett. 41 (2005), pp. 638-640.

[10]T. Geernaert, T. Nasilowski, K. Chah, M. Szpulak, J. Olszewski, G. Statkiewicz, J. Wojcik, K. Poturaj, W. Urbanczyk, M. Becker, M. Rothhardt, H. Bartelt, F. Berghmans, H. Thienpont: Fiber Bragg Gratings in Germanium-doped Highly-Birefringent Microstructured Optical Fibers, IEEE Photonics Technology Letters 20 (8), 554-556 (2008).

[11] M. Becker, J. Bergmann, S. Brueckner, M. Franke, E. Lindner, M. Rothhardt and H. Bartelt, Fiber Bragg grating inscription combining DUV sub-picosecond laser pulses and two-beam interferometry. Optics Express 16(23) (2008), 19169.

[12]Y. Wang, H. Bartelt, W. Ecke, R. Willsch, J. Kobelke, M. Kautz, S. Brueckner, and M. Rothhardt: Fiber Bragg Gratings in Small-Core Ge-Doped Photonic Crystal Fibers. Journal of Electronic Science and Technology of China 6(4) (2008), 1. 